\title{
Oportunidades de participación étnica en América Latina - Una nueva evaluación contextual con referencia a Bolivia, Colombia y Nicaragua
}

\author{
Marie-Sophie Heinelt \\ FernUniversität, Hagen, Alemania. \\ Email: Marie-Sophie.Heinelt@fernuni-hagen.de \\ Michael Stoiber \\ FernUniversität, Hagen, Alemania \\ Email: Michael.Stoiber@fernuni-hagen.de
}

\begin{abstract}
Resumen: América Latina es una región de gran actualidad para el análisis de instrumentos participativos para grupos étnicos. Este artículo examina su potencial para una inclusión efectiva de los pueblos indígenas y afrodescendientes. Argumentamos que los instrumentos deben diseñarse de manera apropiada, tomando en cuenta el contexto social del país. Además, es necesario considerar las características organizativas de los pueblos, así como limitaciones estructurales tales como las relaciones de poder. Estudiamos Bolivia, Colombia y Nicaragua, países que utilizan diferentes instrumentos para fomentar la participación étnica: consulta previa, autonomía y cuotas étnicas. Demostramos que estos instrumentos tienen efectos positivos, aunque también detectamos déficits, derivados de su implementación inadecuada y relaciones asimétricas de poder. El estudio contribuye a engrosar la creciente literatura sobre cómo los estados latinoamericanos manejan su composición multiétnica. Nuestros resultados revelan la importancia de mirar más allá de las renovaciones institucionales para promover la autodeterminación indígena y afrodescendiente.
\end{abstract}

Palabras claves: Participación política; pueblos indígenas; mecanismos participativos; inlusión efectiva

\section{Opportunities for ethnic participation in Latin America - A new contextual assessment with reference to Bolivia, Colombia and Nicaragua}

\begin{abstract}
Latin America is a highly topical region for the analysis of participatory tools for ethnic groups. This article examines its potential for effective inclusion of indigenous and Afro-descendent peoples. We argue that the instruments should be designed in an appropriate manner, taking into account the social context of the country. In addition, it is necessary to consider the organizational characteristics of the peoples, as well as structural constraints such as power relations. We studied Bolivia, Colombia and Nicaragua, countries that use different instruments to promote ethnic participation: prior consultation, autonomy and ethnic quotas. We showed that these instruments have positive effects, although we also detected deficits, derived from their inadequate implementation and asymmetric power relations. The study contributes to expand the growing literature on how Latin American states manage their multiethnic composition. Our results reveal the importance of looking beyond institutional renovations to promote indigenous and Afro-descendant self-determination.
\end{abstract}

Keywords: Political participation; Indigenous peoples; participatory mechanisms; effective inclusion

\section{Oportunidades de participação étnica na América Latina - Uma nova avaliação contextual com referência à Bolívia, Colômbia e Nicarágua}

Resumo: A América Latina é uma região de enorme atualidade para a análise de instrumentos participativos para grupos étnicos. Este artigo examina seu potencial para uma inclusão efetiva de povos indígenas e afrodescendentes. Argu- 
mentamos que os instrumentos devem ser projetados adequadamente, levando em consideração o contexto social do país. Além disso, é necessário considerar as características organizacionais dos povos, bem como as limitações estruturais tais como as relações de poder. Estudamos Bolívia, Colômbia e Nicarágua, países que utilizam diferentes instrumentos para promover a participação étnica: consulta prévia, autonomia e cotas étnicas. Demonstramos que esses instrumentos têm efeitos positivos, embora também detectemos déficits, derivados de sua implementação inadequada e relações de poder assimétricas. O estudo contribui para expandir a crescente literatura sobre como os estados latino-americanos lidam com sua composição multiétnica. Nossos resultados revelam a importância de ir além das reformas institucionais para promover a autodeterminação de indígenas e afrodescendentes.

Palavras chave: Participação política; povos indígenas; mecanismos participativos; inclusão efetiva.

$* * *$

\section{Introducción}

América Latina es una región de gran actualidad de cara a instrumentos legales para la participación política de los pueblos indígenas y afrodescendientes. Encontramos diversos mecanismos participativos, tales como la consulta previa, la autonomía y las cuotas étnicas. Evalúamos países paradigmáticos, que cuentan con estos canales de participación étnica, así como sus perspectivas para una inclusión efectiva. En primer lugar, argumentamos que estos instrumentos deben diseñarse de manera apropiada, tomando en consideración las particularidades del contexto social de cada país. Además, nuestro enfoque está impulsado por la hipótesis de que los instrumentos de participación formales por sí mismos no conducen automáticamente a políticas más inclusivas; deben tener también el potencial para ser utilizados efectivamente. Por lo tanto, se examinan a fondo las características organizativas de los pueblos indígenas y afrodescendientes, así como posibles limitaciones estructurales.

Hasta ahora se han estudiado estos instrumentos de participación principalmente según cada caso (Wright y Tomaselli, 2019; González et al., 2010), mas aún no conjuntamente, como ejemplos de un mecanismo compartido. Nuestra evaluación contextual también va mas allá de la focalización en cambios institucionales que ha prevalecido en la literatura e incluye un estudio comparativo de las organizaciones étnicas y limitaciones estructurales tales como las relaciones de poder, que resultan centrales para entender las diferencias en el grado de éxito de los procesos participativos.

En un primer paso, presentamos el modelo contextual (Abromeit, 2004; Stoiber, 2011). Este marco teórico se aplica para evaluar el entorno institucional de Bolivia, Colombia y Nicaragua, tres países que ofrecen instrumentos típicos para la participación étnica en América Latina. En un segundo paso, integramos otras variables para determinar la efectividad de la participación: examinamos si los pueblos indígenas y afrodescendientes pueden usar los nuevos instrumentos para participar de manera efectiva; para nuestra evaluación será crucial la integración de aquellos factores que podrían impedir que un entorno institucional apropiado se haga efectivo. Para ello, examinamos la capacidad organizativa y una serie de limitaciones estructurales (por ejemplo, las relaciones de poder).

En conclusión, sobre la base de una síntesis comparativa de los tres estudios de caso, demostramos que los instrumentos legales orientados a la participación de los pueblos indígenas y afrodescendientes pueden tener efectos positivos; pero también detectamos déficits cuando se trata de su uso práctico, básicamente derivados de una implementación inadecuada y de restricciones informales. Terminamos con una breve reflexión sobre el riesgo de alimentar conflictos interétnicos cuando los países experimentan con diseños participativos incipientes.

\section{El contexto social y la participación étnica - Marco teórico}

Existe un interés mundial en aumentar la participación ciudadana, en democracias tanto establecidas como nuevas. En las últimas dos décadas, la implementación de instrumentos de democracia directa y deliberativa ha avanzado a nivel mundial (Smith, 2009; Faletti y Riofrancos, 2018). Sin embargo, emanando de diagnósticos sobre estructuras ineficaces, encontramos una discusión activa sobre si más instrumentos participativos pue- 
den realmente resolver los déficits democráticos (Fishkin, 2009; Newton y Geissel, 2011). Nos basamos en esta discusión y argumentamos que más oportunidades de participación no conducen automáticamente a políticas más inclusivas.

La teoría de la democracia ofrece dos enfoques para deducir del contexto un conjunto adecuado de instrumentos participativos. De un lado, tenemos un enfoque que se centra en la desigualdad social, la misma que puede amenazar la estabilidad de las democracias. De otro lado, el segundo enfoque se centra en la segmentación social que puede imponer ciertas demandas sobre el diseño institucional de una democracia estable. Estos enfoques se presentan con más detalle en los párrafos siguientes. Integramos ambos elementos y nos adherimos al argumento básico de que las características del contexto social pueden amenazar la democracia mayoritaria. Proponemos un concepto de democracia liberal. Según este concepto, «la democracia busca asegurar la libertad individual y la igualdad. Es la extensión de la autodeterminación individual en el ámbito de las decisiones colectivas; es promulgada por individuos que participan efectivamente en las decisiones que les afectan» (Stoiber, 2011, p. 113; traducido por los autores). ${ }^{1}$

El razonamiento de Kymlicka (1995) a favor de los derechos colectivos se vincula directamente con la comprensión de la democracia que hemos elegido. Kymlicka argumenta que la libertad individual puede reconciliarse con la concesión de derechos colectivos para las minorías étnicas ${ }^{2}$ y construye su argumento a favor de los derechos colectivos sobre los principios de igualdad y libertad. La principal preocupación de Kymlicka es cómo conservar la cultura de un grupo en el marco general del Estado. Kymlicka propugna la representación minoritaria especial ante las presiones externas creadas por el predominio de los grupos mayoritarios; en consecuencia, en lo que respecta a las reglas electorales sugiere un sistema de votación proporcional, con un umbral de representación lo más bajo posible para mejorar la igualdad en el proceso político. Sin embargo, tal igualdad formal de oportunidades a menudo puede ser insuficiente, ya que las minorías con frecuencia padecen al mismo tiempo desventajas económicas. Es por eso que, según Kymlicka, dichas desventajas, profundamente arraigadas, justifican la representación minoritaria garantizada, por ejemplo, a través de un número fijo de escaños legislativos (ibíd).

Además, para garantizar la autodeterminación de las minorías, recomienda dos mecanismos: diferentes formas de autonomía y derechos de veto. Si los grupos minoritarios conforman una mayoría regional, una solución ideal sería la autonomía territorial (ibíd.). Si no están concentrados territorialmente, las áreas de toma de decisiones autónomas podrían asegurar el autogobierno sobre un tema específico como forma de autonomía funcional. La autonomía funcional abarca campos de políticas que son centrales para la identidad cultural del grupo (como la educación). Los derechos de veto son un instrumento alternativo para proteger los intereses minoritarios en estos campos.

Lijphart (1999) extiende el enfoque de la inclusión de minorías a las sociedades segmentadas. La segmentación social existe cuando coexisten grupos distintos cuyas demandas están más orientadas a la separación que a la interdependencia. Lijphart acuñó el ideal normativo de la democracia de concordancia, que se basa en compartir el poder y en la participación de todos los grupos relevantes. Por consiguiente, en primer lugar, en las sociedades segmentadas, sistemas de mayorías calificadas deberían reemplazar un sistema de voto por mayoría cuando el consenso social básico se ve afectado. Además, Lijphart argumenta que la representación proporcional y las grandes coaliciones deberían complementarse otorgando autonomía o derechos de veto, si las políticas son sensibles para algunos grupos étnicos. Por lo tanto, y en esto se asemeja al razonamiento de Kymlicka, la autonomía y los derechos de veto deben estar reservados en un contexto con minorías étnicas, mientras que para las sociedades segmentadas se consideran suficientes las mayorías calificadas. La idea de Lijphart de dimensiones de campos políticas (issue-dimensions) es además útil para identificar líneas de conflictos ideológicos en sociedades segmentadas, lo que va de la mano con el concepto de Lipset (1959) sobre las estructuras de clivajes. Sobre la base de estos conceptos, los conflictos regionales entre el centro y la periferia de un país también son características relevantes, que podrían resolverse mediante los derechos de autonomía para la periferia (Lijphart, 2012). ${ }^{3}$

La desigualdad socioeconómica es un tema aún más importante para la calidad de la democracia en América Latina. De acuerdo con Dahl (1989), la distribución equitativa de los recursos es una condición necesa- 
ria para la igualdad de oportunidades en el proceso político: «Gaining enlightened understanding» también es identificado como una condición previa para el proceso democrático, que debe basarse en «adequate and equal opportunities for discovering and validating [...] the choice on the matter to be decided that would best serve the citizens' interest» (Dahl, 1989, p. 112). Dahl (1996) sostiene que la igualdad de oportunidades para la participación depende del acceso a los recursos socioeconómicos. Por consiguiente, la desigualdad socioeconómica distorsiona la igualdad política.

En conclusión, en combinación con la segmentación social, la desigualdad se convierte en un elemento importante a considerar cuando se investiga el diseño de instrumentos de participación. De la combinación de segmentación social y desigualdad, es decir, de situaciones en las que grupos socioeconómicamente desfavorecidos son al mismo tiempo segmentos cerrados o minorías étnicas, como en América Latina, surge una situación particularmente delicada. Tal superposición puede tener un efecto de refuerzo y pueden surgir serios problemas para una participación efectiva.

\section{Figura 1}

\section{Contexto social e instrumentos requeridos}

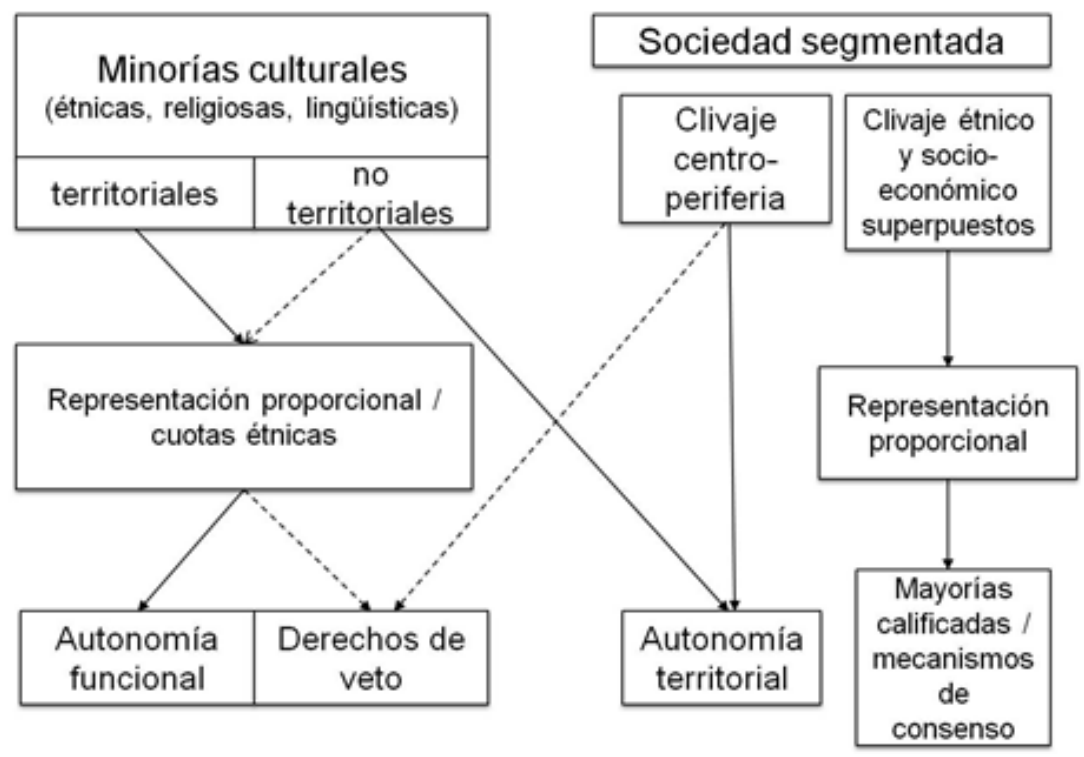

Fuente: Elaboración propia.

En la figura 1 se presentan las configuraciones factibles del contexto social e instrumentos apropiados. La interdependencia entre instrumentos representativos y alternativos para una participación más directa se hace evidente. Primero, si hay pueblos indígenas y afrodescendientes en un país, examinamos si están concentrados territorialmente. Si no lo están, la representación garantizada en la toma de decisiones a nivel nacional puede ser suficiente. Si los grupos comparten una fuerte identidad étnica, también vemos la necesidad de autonomía funcional en aquellos campos políticos que conciernen a su identidad cultural. Los derechos de veto también pueden configurarse como una solución (cualitativamente más débil) para dichos campos. Si un grupo está concentrado territorialmente, según nuestro modelo la autonomía territorial, es un instrumento suficiente para salvaguardar su autodeterminación. ${ }^{4}$ Una segunda solución para el mismo contexto sería por analogía con grupos no territoriales.

En sociedades segmentadas, los conflictos ideológicos a menudo atraviesan un país. Aquí, solo la división centro-periferia se encuentra en un eje territorial. En tal contexto, la participación también puede fomentarse mediante la autonomía territorial. Los derechos de veto una vez más deben ser vistos como segunda 
mejor solución debido a su orientación basada en el status quo. Si encontramos un contexto con varios conflictos ideológicos (es decir, si, por ejemplo, las divisiones étnicas y socioeconómicas se superponen) según el modelo la participación puede ocurrir sobre la base de la representación proporcional, aunque no sea suficiente por completo. Para obtener el consentimiento necesario, se necesitarían además mayorías calificadas y mecanismos de consenso para los procesos de toma de decisiones.

\section{Aplicación del modelo Métodos y diseño de los estudios de caso}

Buscamos probar si los instrumentos de participación étnica que encontramos en Bolivia, Nicaragua y Colombia pueden ser usados de manera efectiva. Siempre de acuerdo con el modelo, comenzamos por examinar el contexto social de cada país con el objeto de deducir conjuntos ideales de instrumentos participativos y mecanismos de consenso. Los siguientes criterios se utilizan como dimensiones para calificar el contexto social: el grado de segmentación, la concentración regional de grupos étnicos y la segmentación a lo largo de clivajes sociales.

El estudio se basa en un análisis cualitativo del contenido de documentos oficiales (por ejemplo, resultados electorales y expedientes de procedimientos legales), de prensa y de fuentes secundarias. Después de deducir cuáles son los instrumentos para cada país, los examinamos y analizamos si pueden ser usados efectivamente. Los siguientes elementos serán considerados para este paso: (a) el diseño exacto de los instrumentos de participación étnica para calificar la efectividad institucional; es decir, si los instrumentos participativos se implementan de manera tal que los grupos abordados pueden usarlos efectivamente; (b) las relaciones de poder y sus características en relación con la segmentación social: ¿en qué medida podrían las relaciones de poder frustrar el acceso a la toma de decisiones? Para América Latina, en particular, se ha demostrado que las estructuras informales de poder restringen fuertemente la efectividad de las regulaciones formales (Levitsky, 2012; Montambeault y Goirand, 2016); (c) las características organizativas de los pueblos indígenas y afrodescendientes, que constituyen otra variable importante. Sin organizaciones intermediarias coherentes será difícil hacer uso de instrumentos participativos.

Para la selección de estudios de caso, elegimos una N pequeña de países paradigmáticos que han codificado diferentes instrumentos de participación étnica. En consonancia con la figura 1, hemos elegido países con autonomía territorial (Bolivia, Nicaragua), con representación étnica electoral garantizada (Colombia) y con consultas previas como ejemplos de autonomía funcional (Colombia, Bolivia). Con base en esta muestra, analizamos un conjunto de casos más probables (most-likely cases) para la participación étnica en América Latina. Examinamos dichos casos, que representan diferentes combinaciones de las variables intermedias que puedan impedir que instrumentos de participación étnica se hagan efectivos, mediante el metodo de análisis de casos (within-case analysis) (Goertz, 2017).

\section{El contexto social y la participación étnica en Bolivia, Colombia y Nicaragua}

Con respecto a su grado de segmentación étnica, los tres países pueden ser situados en un continuo: en Bolivia, una parte importante de la población es indígena (62 \%). Los pueblos indígenas bolivianos se dividen en varios grupos y hay fuertes facciones intergrupales que discurren paralelamente a la división regional entre las Tierras Altas (principalmente Aymara) y las Tierras Bajas, donde viven grupos indígenas más diversos y pequeños (INE, 2012). Nicaragua y Colombia tienen una proporción mucho menor de pueblos indígenas. En Colombia, el 3.4\% de la población es indígena y se divide en más de 80 pueblos diferentes. El porcentaje de afrocolombianos (10.4\%) hace de Colombia el país con la segunda población afrodescendiente más grande de la región, después de Brasil (DANE, 2005). El $8.5 \%$ de la población nicaragüense se identifica como miembros de un grupo étnico que no solo incluye a los indígenas, sino también a una pequeña proporción de afronicaragüenses (Garífunas y Kriol) (INEC, 2005). En los tres casos, el grado de concentración territorial de los grupos también varía, aunque en cada país encontramos grupos que constituyen mayorías regionales. En Bolivia, los departamentos andinos están habitados principalmente por pueblos indígenas, y los mestizos de origen europeo están 
más representados en las Tierras Bajas. Los indígenas constituyen mayorías en cinco departamentos (Potosí, Oruro, Cochabamba, La Paz, Chuquisaca; Cepal, 2001) y más del 60 \% de los municipios del país tiene mayorías indígenas (Colque, 2009). En Colombia, los indígenas están más dispersos y en su mayoría residen en comunidades monoétnicas en áreas remotas. Una gran parte vive en la región suroeste. En el departamento de Amazonas, escasamente poblado, constituyen la población mayoritaria (DANE, 2005). Los afrocolombianos se concentran en la costa del Pacífico; en el departamento de Chocó representan el 90 \% de la población (ibíd). En Nicaragua, los grupos étnicos viven principalmente en la periferia rural (INEC, 2005). Dependiendo de sus raíces históricas, los indígenas viven en la costa, ya sea del Pacífico o del Caribe. Hoy en día, a nivel departamental ya no conforman mayorías, aunque están altamente concentrados en la costa del Caribe. En la RAAN (Región Autónoma del Atlántico Norte) nicaragüense, los Miskitos siguen constituyendo mayorías en algunos municipios (ibíd).

De los tres países, la segmentación social es más compleja en Bolivia, donde las divisiones sociales se superponen a muchos niveles. Desde la década de 1980, la identidad indígena se ha polítizado cada vez más (Flesken, 2013). Al igual que en Colombia, las organizaciones indígenas se han creado para defender demandas que van más allá del reconocimiento cultural y se orientan también a lograr mejoras en la profunda desigualdad existente y a incidir en temas relacionados con los recursos naturales y la conservación del medio ambiente (Grisaffi, 2010). A principios del siglo XXI se agudizaron las divisiones ideológicas entre la élite blanca y mestiza y el Movimiento al Socialismo (MAS), que representaba a la izquierda en coalición con los movimientos indígenas. Paralelamente, la división regional entre las Tierras Bajas más ricas y dominadas por las élites mestizas, y las Tierras Altas dominadas por la población Aymara, se ha exacerbado con el acceso al gobierno del MAS de Evo Morales (Centellas, 2016). La división regional también discurre paralelamente a la existencia de facciones interétnicas entre distintos grupos indígenas. Para hacerlo aún más complejo, los grupos indígenas de las Tierras Bajas están organizados de una manera heterogénea y las relaciones entre los distintos grupos son tensas (Alderman, 2018). ${ }^{5}$

En Colombia, la coalición del Frente Nacional, el sistema bipartidista entre partidos liberales y conservadores, terminó a principios la década de 1990 (Pizarro, 2006) cuando se hicieron intentos para abrir este sistema dualista que había excluido a otros actores políticos durante décadas (Kastner, 2015). Las organizaciones indígenas se beneficiaron de las respectivas reformas electorales que se llevaron a cabo en la década de 1990. Desde fines de los años 1960, organizaciones como el Consejo Regional Indígena del Cauca (CRIC) comenzaron a movilizarse a nivel regional y, a fines la década de 1970, varias organizaciones ya lograron actuar relativamente unidas a nivel nacional. A pesar de su bajo número, los pueblos indígenas colombianos crearon organizaciones estables para la representación nacional, como por ejemplo la Organización Nacional Indígena de Colombia (ONIC) (Rice y Van Cott, 2006). ${ }^{6}$ Aunque los afrocolombianos también lograron crear organizaciones nacionales, por ejemplo Cimarrón, la política nacional de blanqueamiento cultural impidió el surgimiento de una identidad política afrocolombiana (Wade, 2010). En general, la segmentación étnica discurre paralelamente a los fuertes patrones regionales de desigualdad en Colombia. Los indígenas y afrodescendientes pertenecen a los segmentos más desfavorecidos y viven en las zonas más pobres y periféricas.

Nicaragua no es solo uno de los países más pobres de América Latina. También está muy marcado por una fuerte desintegración centro-periferia y una notable desigualdad económica entre la capital y áreas más distantes (INEC, 2005). En 2007, el ex partido revolucionario Frente Sandinista de Liberación Nacional (FSLN) tomó el gobierno. Hoy, el presidente Daniel Ortega ha estabilizado su posición dominante mediante una mezcla de reformas institucionales, fraude electoral y represión (Thaler, 2017; Martí i Puig, 2015). En años anteriores, el FSLN consiguió el apoyo de los grupos marginados, y fuerzas de oposición como el Partido Liberal Constitucionalista (PLC) hasta hoy son débiles (Peréz-Baltodano, 2012). La relación de los pueblos indígenas con el FSLN es históricamente propensa a conflictos y oscila entre cooperación pragmática y confrontación abierta (Gonzaléz, 2011). Los indígenas nicaragüenses viven en las regiones más pobres del país que están sumamente descuidadas por la administración pública (Téllez, 2009).

Además de los Sumu-Mayangna y los Rama (Bryan 2019; Sylvander, 2018), es el grupo indígena más numeroso de Nicaragua, los Miskitos, el que tiene una influencia política considerable (Barraco, 2019). Su cuerpo representativo es el partido regional Yatama, que es uno de los partidos de conformación étnica más 
antiguos de América Latina y tiene sus orígenes en la organización Misurata fundada en la década de 1970 (Arraiza, 2014).

\section{Instrumentos sugeridos y estructuras reales}

Con respecto a la concentración regional de grupos indígenas en Bolivia, ${ }^{7}$ la figura 1 sugeriría que existe la autonomía territorial. A nivel departamental, esto podría ser factible para Potosí, Oruro, Cochabamba, La Paz y Chuquisaca. Sin embargo, dado que estas áreas no están habitadas mono-étnicamente, su población no puede atribuirse a un grupo indígena mayoritario y la autonomía municipal parece ser una solución más apropiada para los municipios con mayorías indígenas en las Tierras Altas y Tierras Bajas. Una vez más, hay que tener en cuenta asimismo que estos municipios rara vez son monoétnicos. Por lo tanto, los residentes de minorías no indígenas (mestizos y blancos) deberían contar con autonomía funcional en los municipios indígenas entonces autónomos.

Para garantizar la representación de los diversos grupos étnicos a nivel nacional, el mecanismo de la representación proporcional en la Asamblea Nacional parece apropiado. Para reconciliar conflictos en torno a los beneficios de la explotación de los recursos naturales entre las regiones bolivianas (Mähler y Pierskala, 2014), una opción adicional sería establecer un fondo nacional de compensación y ajuste financiero como herramienta de consenso para distribuir los ingresos.

La Constitución Política de 2009 define a Bolivia como un Estado «plurinacional» y contempla cuatro niveles de autonomía: para territorios autónomos regionales, departamentales, municipales e indígenas. La Ley de Autonomías y Descentralización (2010) fundamenta las capacidades y los procesos que involucran a los niveles autónomos: la Autonomía Indígena Originario Campesina (AIOC) se otorga exclusivamente a los pueblos indígenas concentrados territorialmente (Art. 6, II). Los cuatro tipos de autonomía se obtienen a través de un referéndum (Art. 50-54). Para obtener el rango de AIOC, el Ministerio de Autonomías tiene que certificar el carácter ancestral de la unidad territorial (Art. 56). La certificación requiere una prueba de la viabilidad del autogobierno, que se califica mediante una evaluación de la organización política y un plan de desarrollo territorial, así como el cumplimiento de una cuota demográfica mínima. ${ }^{8}$ Cabe destacar que los territorios indígenas pueden traspasar fronteras municipales convencionales para poder adaptarse a la delimitación de las tierras ancestrales. Deben establecer un estatuto autonómico que considere a las minorías no indígenas, otorgándoles derechos constitucionales. Una vez que la autonomía entra en vigor, la administración autónoma puede ejercer poderes legislativos y reguladores que cubren varios campos de las políticas públicas (vivienda, recursos naturales renovables, transporte, etc.) (Art. 45). Todas las unidades autónomas están integradas en el presupuesto del gobierno nacional (Art. 106-131).

La Constitución de 2009 también prevé consultas, que constituyen una forma de autonomía funcional según nuestro modelo, en el caso de medidas legislativas y administrativas y de explotación de los recursos naturales que afectan el territorio indígena (Art. 30, 15). La Ley de Hidrocarburos (Ley 3058) y el Decreto 29033 regulan las consultas en este sector. Con respecto a otras áreas de autonomía funcional, de acuerdo a la Constitución la jurisdicción indígena se encuentra a la par de la jurisdicción regular (Art. 179).

La representación indígena está garantizada en el Senado y en la Cámara de Diputados por cuotas electorales (Art. 147, II). Además, la Constitución prevé el establecimiento de distritos electorales indígenas en zonas donde los pueblos indígenas conforman minorías (Art. 146, VII). Con respecto a la representación de diferentes segmentos sociales, la votación proporcional se utiliza para la Cámara de Diputados (Art. 146, 147). Además, cada departamento tiene cuatro representantes en el Senado que son elegidos en listas (Art. 148).

En resumen, la legislación de Bolivia otorga muchos derechos a su población indígena y también ofrece instrumentos para que los diversos segmentos sociales y regiones estén representados. Sin embargo, los procedimientos nacionales de toma de decisiones carecen de requisitos de consenso suficientes para nivelar las múltiples divisiones que atraviesan el país. Por ejemplo, no existe un requisito para mayorías calificadas en ningún campo de decisiones, como lo sugeriría Lijphart en relación con dicho contexto. 
En Colombia, la concentración territorial de afrocolombianos e indígenas ofrece la opción de autonomía territorial, de acuerdo con nuestro modelo. Sin embargo, incluso después de que el Acuerdo de Paz de 2016 formalmente pone fin a más de 50 años de guerra civil, todavía encontramos un contexto de Estado limitado donde la autoridad pública no llega a todas regiones (Gill, 2017). Como los esfuerzos de autonomía territorial son problemáticos sin una categoría de Estado, la autonomía funcional, así como los mecanismos de consenso, parecen ser formas más apropiadas para garantizar la participación étnica. Además, la representación de todos los segmentos sociales debería apoyarse en un sistema de votación proporcional, complementado por cuotas étnicas.

El parlamento colombiano está formado por dos cámaras donde se aplica la representación proporcional. Algunos asientos están reservados para representantes indígenas y afrocolombianos (República de Colombia, 1991, Art. 171). Desde las reformas electorales de 1991, todos los miembros del Senado son elegidos en un distrito electoral nacional. Una razón de esta reforma fue facilitar el acceso de representantes étnicos al Senado, al permitir que grupos pequeños y dispersos ganen votos en todo el país y obtengan escaños (Pizarro, 2006).

Con respecto a la autonomía funcional, se ha otorgado autonomía administrativa limitada a los territorios indígenas mediante los resguardos, aunque sus competencias permanecen bajo la supervisión de las provincias (Art. 286, 321). Formalmente, en los resguardos, se puede ejercer la ley indígena y se han fortalecido los sistemas educativos indígenas propios (Art. 246) (Herrera, 2019). Sin embargo, el sistema del resguardo se mantiene inestable por un lado debido a la falta de titulación por parte de las instituciones del Estado y, por el otro, debido a la presencia sostenida de fuerzas irregulares (Weitzner, 2019).

El artículo 330 y el decreto 1320 regulan la consulta previa en torno a proyectos y medidas administrativas y legislativas que afectan a los territorios indígenas y afrocolombianos (para este grupo, véase la Ley 70) (LaraLargo, 2019). ${ }^{9}$ En general, los esfuerzos formales orientados a promover la participación étnica parecen apropiados con relación al contexto social en Colombia.

Al desviar nuestra atención a Nicaragua, descubrimos que los grupos indígenas son pequeños y una gran parte reside en áreas dispersas del país. Sin embargo, podrían instaurarse escaños reservados en la Asamblea Nacional para garantizar su representación. Además, se debería otorgar autonomía funcional en los campos de educación y derechos lingüísticos. Para los Miskitos que están concentrados regionalmente, la autonomía territorial es la medida apropiada para salvaguardar su autodeterminación según nuestro modelo. Finalmente, se podría lograr una acomodación del clivaje centro-periferia fortaleciendo las competencias de los departamentos frente a la capital, Managua, a través de la descentralización.

Nicaragua fue uno de los primeros países de América Latina (además de Panamá) que otorgó autonomía a unidades subestatales indígenas. Desde 1987, dos regiones de la costa del Caribe son autónomas (República de Nicaragua, 1987; Estatuto de Autonomía, 1987). Hoy en día, la composición multiétnica de las regiones mencionadas se ha vuelto problemática. En la actualidad, seis grupos étnicos habitan dichas regiones: tres grupos indígenas (Miskitu, Sumu-Mayangna, Rama), dos grupos afrodescendientes (Kriol, Garífunas) y grupos mestizos (González et al., 2010). La Ley de autonomía establece que todos los grupos deberían estar representados en los gobiernos regionales o Consejos Regionales. Estos tienen competencias legislativas y administrativas en varios campos, entre ellos planificación económica, salud, gestión de los recursos naturales y educación. También pueden usar el veto contra proyectos de explotación de recursos naturales planeados desde el Estado central. Debido a la intensa migración de los pobres urbanos a la costa caribeña, actualmente los mestizos dominan los Consejos, a pesar de las cuotas vigentes, que deberían asegurar la representación de todos los grupos étnicos (González, 2018).

En respuesta a la presión internacional, en 2003, el gobierno de Nicaragua aprobó la Ley 445 que regula la demarcación de territorios étnicos colectivos en las regiones del Atlántico. Desde entonces, la creación de unidades semiautónomas a nivel local está asegurada formalmente; la ley amplía las competencias de las asambleas locales de los territorios indígenas y afronicaragüenses, por ejemplo en lo que se refiere a la gestión de los recursos naturales (Art. 16). 
Con respecto al nivel nacional, la educación bilingüe está garantizada constitucionalmente para todos los pueblos indígenas en Nicaragua (Art. 121). Los miembros de la Asamblea Nacional son elegidos sobre la base de 20 distritos electorales nacionales y 70 de los departamentos. Las administraciones departamentales tienen derecho a iniciativas legislativas en la Asamblea, pero el hecho de que dependan económicamente del gobierno central restringe su independencia en gran medida (Hooker, 2010).

\section{La participación étnica en la práctica}

En esta sección proporcionamos una evaluación de cómo se pueden utilizar los instrumentos formales de participación en la práctica. Para ello, se examina en detalle el entorno institucional y las características de las organizaciones intermediarias, así como algunas limitaciones estructurales.

\section{Efectividad institucional}

A primera vista, las disposiciones diseñadas para garantizar para la autonomía en Bolivia parecen ofrecer un enfoque apropiado para igualar la distribución territorial heterogénea y la diversidad de los grupos indígenas. En ese sentido, el hecho de que las AIOC puedan exceder los límites administrativos de los municipios puede destacarse como justo. Además, las competencias de autogobierno, incluida la administración de los recursos económicos, así como el ejercicio de los poderes legislativos y administrativos en campos centrales de la política, parecen fomentar una autodeterminación sustancial de las unidades subnacionales. Hasta hoy, 18 territorios indígenas se han incorporado al proceso legal para obtener la autonomía. Sus esfuerzos han alcanzado diversas etapas del procedimiento de reconocimiento. Más de siete años después de iniciado el proceso de autonomía, tres territorios, Chipaya, Charagua y Raqaypampa, obtuvieron el rango de AIOC. ${ }^{10}$ Actualmente, un territorio, Gutiérrez, todavía se encuentra a la espera de la aprobación de su estatuto por el Tribunal Constitucional. En dos territorios, los residentes suspendieron sus estatutos autonómicos en referendos finales (Totora Marka, Huacaya) (SEA, 2018). En general, las organizaciones indígenas critican las condiciones establecidas en la Ley de Autonomía, aduciendo que presentan demasiados obstáculos para lograr la autonomía (Plata y Cameron, 2017). Primero, varios requisitos se consideran demasiado difíciles de cumplir y algunos criterios arbitrarios se prestan a la interferencia de la administración política (Tockman, 2017). Segundo, en la práctica, el diseño institucional de las unidades autónomas sigue sin ser totalmente adecuado para la gran heterogeneidad del contexto sociocultural (Tomaselli, 2016).

Con respecto al primer punto, el límite de tiempo restrictivo y la falta de apoyo administrativo podrían haber impedido a algunos territorios inscribirse en el proceso de solicitud de autonomía desde el principio. Otros requisitos formales, como la correspondencia entre las tierras ancestrales y el territorio habitado actualmente, así como la necesidad de probar que se cuenta con una administración territorial viable, tampoco son fáciles de cumplir (Tockman, 2014). Por todo ello, el proceso de implementación es de larga duración. En la etapa final, el Tribunal Constitucional tiene que volver a verificar la constitucionalidad de los estatutos autónomicos, lo que puede llevar hasta tres años. En general, el riesgo de quedar entrampados en el proceso legal puede generar frustración e intensificar los conflictos en el entorno multiétnico de Bolivia. Con respecto al segundo punto, las primeras experiencias también demuestran que los pueblos indígenas de las Tierras Bajas que habitan en áreas dispersas han enfrentado dificultades para cumplir con la cuota mínima de residentes (Laing, 2020).

En cuanto a Colombia, lo más notable es que en este país encontramos instrumentos para asegurar la representación legislativa de delegados indígenas y afrocolombianos. La ingeniería electoral (electoral engineering), que abarca la reserva de escaños y distritos electorales especiales para representantes indígenas y afrocolombianos en las dos cámaras legislativas, es comúnmente aclamada por haber fomentado sustancialmente la participación de organizaciones étnicas en la política nacional (Van Cott, 2005). Sin embargo, a segunda vista, nuestros hallazgos sobre la representación actual de indígenas y afrocolombianos en la legislatura muestran una disminución significativa de su presencia: para el período legislativo actual, hay un solo senador afrocolombiano (de un total de 108), y cinco delegados afrocolombianos obtuvieron escaños en la Cámara de Diputados (de un total de 171). ${ }^{11}$ Estos son números bastante pequeños en relación con el porcentaje de afrocolombianos que existen en el país, que es del 10 \%. Curiosamente, solo los dos legisladores que ganaron los distritos electorales 
especiales para los afrocolombianos en la Cámara de Diputados han sido candidatos de organizaciones afrocolombianas. Los representantes afrocolombianos restantes fueron elegidos como candidatos de partidos no étnicos, como Alianza Verde o el Partido Liberal.

Hay dos senadores que obtuvieron sus escaños a través del distrito electoral especial indígena para los partidos indígenas Autoridades Indígenas de Colombia (AICO) y Movimiento Indígena Alternativo y Social (MAIS). En la legislatura actual, dos delegados indígenas se incorporaron a la Cámara de Diputados, representando también al MAIS (Consejo Electoral Nacional, 2018).

Vale la pena analizar el bajo número de representantes indígenas y afrodescendientes en el poder legislativo colombiano. Esta observación contrasta con el notable aumento de los partidos étnicos, y particularmente indígenas, después del proceso constitucional de 1991 y de las reformas multiculturales de la década de 1990 (Lara-Largos, 2019). Reformas electorales más recientes explican el fenómeno en parte: para reducir la fragmentación de los partidos, el Acto Legislativo 19 del 2003 introdujo una cuota mínima del 2 \% de la votación para entrar al Congreso y registrarse como partido político (Laurent, 2010). Este umbral golpeó duramente a las organizaciones étnicas y, en particular, muchas organizaciones indígenas perdieron su estatus legal como partido.

En el caso de Nicaragua, que tratamos a continuación, estamos particularmente interesados en las autonomías de la Costa Atlántica para el autogobierno miskito-indígena. El predominio mestizo en los Consejos Regionales ha creado severos conflictos interétnicos durante las últimas dos décadas y ha debilitado la autonomía costera (Gónzalez, 2018). Esto es particularmente destacable en la RAAS (Región Autónoma del Atlántico Sur), donde los Miskitos todavía constituyen un porcentaje importante de la población (60 \%). En la RAAS, el FSLN domina el Consejo: actualmente, Yatama apenas tiene uno de los siete escaños en la Junta Ejecutiva y el FSLN tiene cinco (quedando un último escaño para el Partido Liberal Constitutionalista [PLC]). ${ }^{12}$ En las elecciones regionales de 2019, Yatama también permaneció relativamente poco representada en la RAAN (Región Autónoma del Atlántico Norte), con $25 \%$ de los votos, contra el 56 \% obtenido por el FSLN (y el 6 \% obtenido por el PLC) (CSE, 2019) ${ }^{13}$ El predominio de los partidos tradicionales, y en particular la hegemonía del FSLN, aún debilitan la influencia de los intereses de los Miskitos de cara al gobierno central. Asimismo, los intentos de Yatama por obtener escaños en la Asamblea Nacional se han visto frustrados. Debido a la imposición de nuevas restricciones a la inscripción de partidos para las elecciones nacionales, desde 2006 Yatama tuvo que formar alianzas electorales y optó por apoyar al FSLN hasta 2014, cuando acusó al FSLN de fraude electoral (González, 2016).

Con la finalidad de subsanar las deficiencias institucionales de las regiones autónomas, en 2003 se estableció un marco legal modificado para fundamentar el autogobierno subregional. La Ley de Tierras Comunales (Ley 445) regula la titulación de las comunidades étnicas y reconoce sus propias formas de gobierno y autoridades. Estas autoridades ahora tienen que otorgar permiso para el uso de los recursos que se encuentran dentro de su territorio. Aunque la ley ha sido acogida como un mecanismo prometedor para fomentar el autogobierno indígena en el actual entorno multiétnico de la Costa Atlántica, el proceso de titulación ha demorado mucho, entre otras cosas debido a la falta de voluntad política; además el proceso ha exacerbado conflictos territoriales que en el pasado habían permanecido latentes (Sylvander, 2018). ${ }^{14}$

\section{Relaciones de poder}

Como ya hemos mencionado, los dilatados avances del proceso de autonomía en Bolivia son atribuidos a un tratamiento arbitrario por parte de los funcionarios públicos (Tomaselli, 2016). El proceso de evaluación y certificación del Ministerio de Autonomía es especialmente propenso a las intromisiones (Lacroix, 2012). Se han reportado intentos de cooptación y presiones a solicitantes indígenas para fortalecer la agenda corporativa del MAS (Fontana, 2014; Thede, 2011). Otro tema es la falta de recursos financieros necesarios para que el Ministerio de Autonomías pueda apoyar técnica- y jurídicamente a los municipios indígenas (Plata y Cameron, 2017).

Otras limitaciones estructurales se dan particularmente en el campo de los recursos naturales. Por ejemplo, el poder de toma de decisiones de las AIOC termina cuando el tema tiene que ver con la gestión de recursos no renovables. Se trata de una limitación crítica, ya que el control completo sobre los recursos naturales constituyerequisito indispensable para la independencia financiera de las unidades subnacionales. A medida 
que los gobiernos de América Latina promueven la extracción de recursos, incluida la administración de Morales (Marston y Kennemore, 2019), surgen graves conflictos en el campo de la gestión de los recursos naturales, tal como lo ilustra el caso TIPNIS. ${ }^{15}$ Es en ese mismo campo de políticas públicas, altamente disputado, en el que las consultas con las comunidades indígenas están legalmente prescritas en Bolivia. Formalmente, estos procedimientos pueden considerarse como un amparo de la autonomía funcional. Sin embargo, en la práctica, los actores corporativos y estatales a menudo ignoran la exigencia legal de consulta (Rodrigues, 2010) o, muchas veces, los procedimientos de consulta se quedan cortos en términos de criterios deliberativos sustanciales (Eichler, 2018). Un defecto común es que los grupos consultados a menudo no reciben información suficiente sobre el proyecto propuesto (Schilling-Vacaflor, 2013). Otro déficit es el carácter no vinculante de los acuerdos tomados como resultado de los procesos de consulta. La falta de competencias de veto ha sido identificada como una de las limitaciones más importantes a la participación indígena efectiva mediante consultas (Fontana, 2013).

En Nicaragua, la asimetría de poder entre el gobierno central y la periferia se ha visto agravada por la hegemonía que ostenta el FSLN desde 2006 (Perez-Baltodano, 2012). Las regiones autónomas se ven especialmente afectadas por la negligencia financiera del gobierno. Pertenecen a las partes más pobres del país con una infraestructura precaria y la tasa dede desempleo más alta (Stocks, 2005; INEC, 2005). Dado que los Consejos Regionales dependen en gran medida de la asignación de fondos de parte del gobierno central, el gobierno nacional puede hacer que los gobiernos regionales sean inoperables e inducir a la cooptación política asignando presupuestos menores o reteniendo los fondos por completo (Hooker, 2009). Desde la remontada del FSLN, la lealtad al partido hegemónico se ha vuelto cada vez más importante (Thaler, 2017; Martí i Puig, 2011).

Una interferencia más directa en la política regional se manifiesta particularmente en el campo de la política de recursos naturales, donde las competencias autónomas frecuentemente se pasan por alto. Dado que formalmente la gestión de los recursos naturales es responsabilidad de los Consejos Regionales, las concesiones también deberían ser autorizadas por los Consejos. Sin embargo, en la práctica, es el gobierno central el que otorga las concesiones (Henrikson, 2008). En la última década se ha acelerado la actividad extractiva sin el consentimiento de los Consejos Regionales y a raíz del desplazamiento de los residentes locales (Navas et al., 2018).

\section{Características organizacionales}

En el caso de Colombia, hemos visto que en la actualidad los representantes afrodescendientes son relativamente más exitosos en obtener escaños legislativos que los candidatos indígenas. Laurent (2010) encuentra una explicación para el debilitamiento de los partidos indígenas en el dilema clásico que enfrentan los movimientos sociales ascendentes. La institucionalización de los movimientos indígenas durante la década de 1990 contribuyó a reforzar luchas internas de poder y liderazgo y acentuó disputas intraorganizacionales. Un asunto crítico de controversia, que afectó particularmente la cohesión interna de las organizaciones tradicionales ASI y AICO, fueron las disputas sobre hasta qué punto deberían ser integrados los grupos sociales afiliados (no indígenas), por ejemplo los campesinos (Jaramillo, 2012).

Como ha demonstrado el conflicto del TIPNIS, las cuestiones relacionadas con el uso de la tierra y los recursos naturales pueden desatar conflictos sobre todo en un movimiento indígena ya de por sí dividido (Canessa, 2018; Anthias, 2018). Las divisiones entre los propios grupos indígenas se han evidenciado como uno de los grandes desafíos que enfrenta la implementación de los cambios institutionales en Bolivia. Se manifestaron en el TIPNIS con respecto al instrumento de la consulta (Alberti, 2019). El proceso de autonomía exacerba asimismo la fragmentación indígena y las luchas de poder locales (Alderman, 2018). Algunos referendos locales sobre las AIOC no solo resultaron en un «no», sino que también en varios territorios los votos a favor del «sí» apenas alcanzaron un poco más del 50\% (CONADETI, 2018). Esto revela una discordancia local e intra-étnica en torno al tema de la autonomía (Fontana y Grugel, 2016). El paso de establecer un estatuto autonómico ha demostrado ser aún más susceptible a los bloqueos internos (Mejillones y Guarachi, 2012). La necesidad de deliberar sobre un estatuto común en algunos casos llevó a una erupción de luchas de poder locales entre diferentes federaciones indígenas y campesinas (Tockman et al., 2015). Además, el requisito de la titulación definitiva de las tierras genera conflictos, intensifica las estructuras de competencia y exacerba las tensiones intra- e intercomunitarias que antes habían estado latentes (Augsburger y Haber, 2018; Alderman, 2018). 
En Nicaragua, una dinámica análoga surgió en el transcurso de la implementación de los nuevos derechos en materia de tierras comunales. Los procesos de demarcación también resultaron ser altamente propensos a los conflictos. Las disputas en torno a la tierrase han intensificado; en algunas disputas se hizo uso dela fuerza (Navas et al., 2018).

Las divisiones existentes entre los propios indígenas como uno de los desafíos para la implementación de los cambios legales también se manifiestan en las elecciones de la RAAN y la RAAS y el predominio del FSLN, derrotando decisivamente a Yatama y reduciéndolo a una fuerza marginal dentro de los Consejos. Según la interpretación de Barraco (2018), debido a las sucesivas revisiones de las leyes electorales, Yatama no sólo ha perdido gran parte de su identidad indígena, sino que también se ha visto en la necesidad de participar en pactos electorales con el FSLN y el PLC, que han utilizado estas alianzas para mejorar su propia fortuna electoral a nivel nacional (González, 2011). ${ }^{16}$

Para completar el cuadro, también hay observaciones positivas que revelan cómo las organizaciones étnicas pueden utilizar los instrumentos de participación incluso en un contexto desfavorable. Yatama ha sido un ejemplo de esto durante mucho tiempo. En años anteriores, sus candidatos ganaron el cargo de gobernador de la RAAS. También hay delegados destacados de los Miskitos que obtuvieron presencia nacional, tras candidaturas regionales con Yatama: Evelin Taylor ha sido representante en la Asamblea Nacional y gobernadora de la RAAN y el fundador de Yatama, Brooklyn Rivera, tuvo un escaño en la Asamblea durante muchos años.

También se ha demostrado, en el caso de Bolivia que los grupos indígenas bien organizados pueden provocar mejoras en las prácticas de consulta deficientes, por ejemplo, exigiendo información adecuada sobre proyectos y adhesión al procedimiento legal (Falleti y Riofrancos, 2018; Machado et al., 2017).

\section{Síntesis comparativa y conclusiones}

El presente artículo ha abordado un modelo deducido de la teoría de la democracia en sociedades segmentadas y multiétnicas para analizar la participación étnica en tres países latinoamericanos que reflejan diferentes procesos políticos. Si bien el mencionado enfoque teórico liberal no ha estado exento de crítica en el debate latinoamericano sobre la autodeterminación de los grupos étnicos, consideramos que dicha perspectiva es pertinente a visión general, no solo sobre el estado actual de las iniciativas participativas en esos países sino también sobre los logros y desafíos comunes que entraña la inclusión de los grupos étnicos. Nuestro modelo contextual podría también ser útil para las evaluaciones de más países que practican la participación étnica, como por ejemplo Panamá, Perú, y, más recientemente, Chile.

Si bien encontramos instrumentos innovadores en Bolivia, Colombia y Nicaragua, no podemos concluir que dichos instrumentos mejoren la participación efectiva. En primer lugar, los tres países solo han implementado parcialmente las estructuras adecuadas para el contexto social específico según el modelo sugerido. En cada país, detectamos problemas en el diseño institucional de instrumentos participativos para los pueblos indígenas y afrodescendientes. Especialmente en Bolivia y Nicaragua, la composición multiétnica de las Tierras Bajas y de la Costa Atlántica, respectivamente, eleva la complejidad demográfica hasta el punto de impidir que las normas actuales que regulan la autonomía territorial funcionen de manera efectiva. Esa observación refleja críticas recientes como la de Eichler (2018), quien también constata que muchas estructuras autónomas aceptadas en América Latina no fueron lo suficientemente diversas como para garantizar procesos eficientes (véase también Cameron, 2013). Los problemas señalados en el diseño institucional también pueden interpretarse como síntomas de un limitado reconocimiento de las propias instituciones de los pueblos indígenas y afrodescendientes, con sus funciones y lógicas sustancialmente distintas a las del Estado liberal (Kuppe, 2010). Podría extraerse como conclusión de dicha crítica que el ejercicio de la autodeterminación y de un multiculturalismo auténtico implicaría transformaciones en la organización del Estado-nación en sí mismo (articulada como más prominente por Hale, 2004). Queda subrayar la necesidad de armonizar y coordinar el sistema estatal de una mejor manera con los propios sistemas de los grupos étnicos. 
Además revelamos una serie de contradicciones legales, sobre todo cuando las competencias se comparten entre el gobierno central y unidades subnacionales. En general, la fuerte dependencia presupuestaria de los gobiernos centrales dificulta el uso efectivo de los acuerdos de autonomía, especialmente en el campo de la gobernanza de los recursos naturales (como vemos en Nicaragua). En Bolivia encontramos altos umbrales formales para establecer la autonomía. En estudios futuros se realizará una evaluación más profunda de si el enfoque boliviano de autogobierno descentralizado funciona de manera efectiva, ya que las primeras entidades autónomas recién han sido fundadas. En Colombia, el umbral del 2 \% para la legislatura tuvo un impacto negativo en la representación indígena y afrodescendiente en el parlamento. La principal restricción legal de la consulta previa es su carácter no vinculante. Otros déficits procesales, por ejemplo, con respecto a información adecuada sobre los proyectos, a menudo cimentan los desequilibrios de poder entre los grupos consultados y los proponentes de los proyectos. Finalmente, nuestro análisis de Bolivia y Nicaragua ha demostrado que el establecimiento de nuevos derechos colectivos, por ejemplo el autogobierno local, pueden intensificar los conflictos (anteriormente latentes), especialmente en su etapa inicial de implementación (véase también Alderman, 2018).

En una segunda dimensión, mostramos cómo las relaciones de poder restringen la efectividad incluso de mecanismos formalmente apropiados. De esta manera, ponemos los reflectores sobre un problema crucial para la participación étnica, el mismo que sigue siendo una limitación para muchas innovaciones democráticas en América Latina (Baiocchi y Ganuzza, 2014; Welp y Milanese, 2018). Demostramos, especialmente en el caso de Nicaragua y Bolivia, como la cooptación por parte del gobierno hace perdurar relaciones asimétricas entre los grupos indígenas y el Estado.

Más allá de ello, revelamos que las organizaciones étnicas no siempre pueden hacer uso de los instrumentos participativos. A menudo, facciones intra- e interétnicas lo impiden. Si no hay un consenso dentro de los grupos, y un grado mínimo de cooperación entre organizaciones, sus recursos colectivos siguen siendo demasiado débiles frente al Estado central o a los actores corporativos. Algunas organizaciones indígenas y afrodescendientes enfrentan enormes dificultades para movilizar a sus miembros. Esto lo observamos particularmente a nivel nacional, mientras que su representación política es más coherente a nivel regional y local (véase también Lucero, 2008). Por un lado, esta observación concuerda con la de Cameron (2013), según el cual la creación de espacios autónomos asumió cierta homogeneidad cultural y política entre los grupos étnicos, lo que no siempre se da. A su vez, obstaculiza los intentos de los grupos de fundamentar su autodeterminación (Alderman, 2018). Por otro lado, especialmente en el ámbito electoral, las organizaciones étnicas enfrentan dificultades a raíz de un sistema individualista y competitivo que presenta exigencias políticas y burocráticas y proyecta a los ciudadanos como individuos y no como unidades colectivas con prácticas tradicionales diferentes. Becker (2015) y otros destacan esta paradoja como el desafío más importante para la participación real de los pueblos indígenas (Van Cott, 2007).

Finalmente, la efectividad de la participación étnica parece estar altamente sujeta al tipo de campo de política (policies). Si hay políticas distributivas en juego (Lowi, 1972), como el manejo de los recursos naturales, frecuentemente la efectividad de los instrumentos participativos se ve desafiada. Esto seguirá siendo una limitación crítica para la participación de los pueblos indígenas y afrodescendientes, ya que los conflictos en torno a los recursos naturales continúan siendo uno de los campos de disputa más prevalentes en la región (Zaremberg y Torres, 2018).

\section{Notas}

\footnotetext{
${ }^{1}$ Reconocemos que la aplicación de un concepto liberal a la participación étnica ha sido criticada (Gaitán-Barrera y Khalil, 2015) por no concordar con la propia concepción indígena de su autodeterminación y empoderamiento colectivo (Hale, 2004; Becker, 2015). Tendremos en cuenta dicha crítica a medida que avanzamos, tanto en la discusión teórica como en el análisis empírico.

${ }^{2}$ El término «minorías» fue criticado sobre todo por ignorar la diferencia entre los derechos de las minorías culturales y el derecho de autodeterminación de los pueblos indígenas (Burguete Cal y Mayor

, 2010). Mientras usemos dicho término para diferenciar a los grupos sociales, por razones de coherencia con las ideas básicas de Kymlicka y Lijphart, para el análisis tendremos en cuenta el objetivo de realizar la autodeterminación de los pueblos indígenas y afrodescendientes.
} 
${ }^{3}$ Lijphart ha aplicado sus ideas a la región latinoamericana en varias ocasiones (Lijphart y Waisman, 2018; Lijphart, 1990).

${ }^{4}$ Las entidades subnacionales autónomas serán representadas a nivel nacional por sus propios delegados. Así, la autonomía y la representación garantizada se complementan.

${ }^{5}$ Véase Canessa (2014) en relación con las complejas concepciones de indigeneidad que se presentan en las Tierras Bajas, con un énfasis especial en las diferencias entre grupos indígenas territoriales y desterritorializados.

${ }^{6}$ Los pueblos indígenas participaron en la Asamblea Constitucional que allanó el camino para la Constitución colombiana de 1991. Esta oportunidad de articular sus propuestas sobre el diseño de la nueva Constitución contribuyó decisivamente a la consolidación de las organizaciones indígenas (Van Cott, 1996). El proceso constitucional también sustentó el multiculturalismo legal en Colombia, por ejemplo mediante la ley 70 que fue derrogada dos años después, en 1993 (Restrepo 2013).

${ }^{7}$ La población indígena es mayoritaria en Bolivia. Sin embargo, a nuestro entender, al dividirse en distintos pueblos indígenas, cada uno de ellos constituye una minoría de la población general boliviana.

${ }^{8}$ El mínimo requerido es de 10.000 residentes para la zona andina y 1.000 residentes para las Tierras Bajas (Art. 58).

${ }^{9}$ Cabe mencionar que la Corte Constitucional ha sido una importante fuerza proactiva, que ha avanzado el derecho a la autodeterminación de los pueblos indígenas y afrodescendientes en sus sentencias. En especial, ha destacado el derecho al consentimiento previo y ha especificado el alcance de los asuntos que deben consultarse (Herrera, 2019).

${ }^{10}$ En 2015, la población de cuatro departamentos (Cochabamba, Chuquisaca, La Paz, Potosí) desaprobó sus estatutos autonómicos, previamente elaborados, en ocasión de referendos finales, mientras que Oruro aprobó su estatuto. En 2017, Gran Chaco fue la primera región en obtener la autonomía (SEA, 2018).

${ }^{11}$ Los datos se refieren al período legislativo comprendido entre 2018 y 2022.

${ }^{12}$ Dos integrantes de la Junta (entre ellos la presidenta del Consejo) son afrodescendientes (ambos del FSLN), mientras que dos son indígenas (del FSLN y Yatama) (Canal 13, 2019). En la RAAS, Yatama solo obtuvo el $5 \%$ de los votos en comparación con el $52 \%$ que obtuvo el FSLN (y el $28 \%$ obtenido por el PLC). Los datos se refieren al período 2019 a 2024 para las elecciones regionales así como, seguidamente, 2016 a 2021 para las elecciones nacionales.

${ }^{13}$ Actualmente, dos de los siete miembros de la Junta Directiva de la RAAN son consejales indígenas (Yatama), mientras que uno pertenece a la etnia Kriol (FSLN) (TN 8, 2019).

${ }^{14}$ Demoró hasta 2017, cuando los 23 territorios que entraron en el proceso de demarcación han sido titulados (CONADETI, 2018).

${ }^{15}$ El conflicto del TIPNIS estalló en 2010, cuando Morales impulsó una carretera que atravesaría el Territorio Indígena y Parque Nacional Isiboro Sécure. Muchos residentes temían que la carretera expusiera la zona a una mayor colonización por parte de cocaleros andinos. Otras comunidades se mostraron satisfechas con las posibilidades que la carretera podría ofrecer (enlazarlas con los mercados, mayor acceso a los sistemas de educación y salud). Tras una marcha de protesta organizada por los opositores a la carretera, Morales se vio obligado a declarar la intangibilidad del parque y a realizar una consulta ex-post. Dicha consulta fue criticada por varias organizaciones, que argumentaron que el MAS había cooptado a los líderes indígenas; sin embargo, según el gobierno la consulta reveló una aprobación de la carretera. Visto globalmente, el conflicto ha llevado a un cuestionamiento de la credibilidad de un gobierno que se presenta a sí mismo como pro indígena (Hirsch, 2019) y ha fracturado el Pacto de Unidad establecido entre varias organizaciones que habían apoyado al gobierno de Morales (Andreucci, 2019).

${ }^{16}$ Los cambios en el entorno electoral, y el pacto de Yatama con el FSLN resultante, llevaron a una tasa de abstención en la Costa Atlántica que alcanzóel 50-60 \% (González, 2016), lo cual sugiere una pérdida significativa de confianza en el marco institucional de la autonomía (Barraco, 2018).

\section{Bibliografía}

Andreucci, D. (2019). Populism, Emancipation, and Environmental Governance. Annals of the American Association of Geographers 109 (2), 624-633.

Arraiza, J. (2014). Squaring Indigenous Circles. International Journal of Minority and Group Rights 19, 69-103

Augsburger, A. y Haber, P. (2018). Visions in conflict. Latin American and Caribbean Ethnic Studies 13

(2), 135--156. 
Baracco, L. (2018). Indigenous Struggles for Autonomy. London: Lexington.

Centellas, M. (2016). The Santa Cruz Autonomía Movement in Bolivia, Ethnopolitics 15 (2), 245-264.

Dahl, R. (1989). Democracy and its Critics. New Haven, USA: Yale University Press.

Eichler, J. (2018). Indigenous intermediaries in prior consultation processes. The Journal of Latin American and Caribbean Anthropology 23 (3), 560-578.

Falleti, T. G. y Riofrancos, T. N. (2018). Endogenous Participation. World Politics 70 (19), 86-121.

Flesken, A. (2013). On the Link between Ethnic Politics and Identification. Ethnopolitics 13 (2), 159-80.

Fontana, L. (2014). Indigenous Peasant ‘Otherness’. Bulletin of Latin American Research 33 (4), 436-451.

Gaitán-Barrera, A. y Azeez, K. (2019). Autonomy in the Carribbean Coast. En L. Baracco (comp.). Lexington.

Goertz, G. (2017). Multimethod Research, Causal Mechanisms, and Case Studies. Oxford: Princeton UP.

Gonzaléz, M. (2018). Governance and Governability: indigenous small-scale fisheries and autonomy in coastal Nicaragua. Maritime Studies 17, 263-273.

Gonzaléz, M. (2008). Governing Multi-ethnic Societies in Latin America. Dissertation: York University.

Hooker, J. (2009). Race and the Politics of Solidarity. Oxford: Oxford UP.

Kymlicka, W. (1995). Multicultural Citizenship. Oxford: Oxford UP.

Laurent, V. (2010). Con bastones de mando o en el tarjetón. Colombia Internacional 71, 35-61.

Lijphart, A. (1999). Patterns of Democracy. New Haven: Yale UP.

Martí I Puig, S. (2015). Social Movements in Nicaragua (1979-2014). En P. Almeida y A. Cordeo (comps.). New York: Springer.

Pizarro, E. (2006). Giants with Feets of Clay: Political Parties in Colombia. En S. Mainwaring et al. (comps.). Stanford: Stanford UP.

Servicio Estatal de Autonomías (SEA) (2018). Desarrollo y Evolución del Proceso Autonómico 2005-2017. Recuperado de http://200.87.110.166/sealinea/linea.html.

Smith, G. (2009). Democratic Innovations. Cambridge: Cambridge UP.

Stoiber, M. (2011). Die Qualität von Demokratien im Vergleich. Baden-Baden: Nomos.

Thaler, K. (2017). Nicaragua. Journal of Democracy 28 (2), 157--169.

Tomaselli, A. (2016). Indigenous Peoples and their Right to Political Participation. Baden-Baden: Nomos.

Wright, C. y Tomaselli, A. (2019). The Prior Consultation of Indigenous Peoples in Latin America. Abingdon: Routledge.

Zaremberg, G. y Torres Wong, M. (2018). Participation on the Edge. Journal of Politics in Latin America 10 (3), 29-58. 